\title{
Determination of the Exciton Formation in Quantum Wells from Time-Resolved Interband Luminescence
}

\author{
J. Szczytko, L. Kappei, J. Berney, F. Morier-Genoud, M.T. Portella-Oberli, and B. Deveaud \\ Institut de Photonique et Electronique Quantiques, Ecole Polytechnique Fédérale de Lausanne (EPFL), \\ CH1015 Lausanne, Switzerland
}

(Received 20 November 2003; published 20 September 2004)

\begin{abstract}
We present the results of a detailed time-resolved luminescence study carried out on a very high quality InGaAs quantum well sample where the contributions at the energy of the exciton and at the band edge can be clearly separated. We perform this experiment with a spectral resolution and a sensitivity of the setup, allowing us to keep the observation of these two separate contributions over a broad range of times and densities. This allows us to directly evidence the exciton formation time, which depends on the density as expected from theory. We also denote the dominant contribution of excitons to the luminescence signal, and the lack of thermodynamical equilibrium at low densities.
\end{abstract}

DOI: 10.1103/PhysRevLett.93.137401

Excitons in quantum wells (QW) form quite an appealing quasiparticle showing a large range of optical properties that have proven at the same time technologically useful, and physically interesting [1]. A large part of this interest is linked with the appearance of excitonic resonances in absorption up to room temperature. It is also well known, since the work of Weisbuch [2], that free excitons appear to dominate the photoluminescence (PL) response of semiconductor $\mathrm{QW}$ at low temperatures. This effect originates in part from the breakdown of the translational symmetry which brings a very efficient recombination channel to free excitons in QWs $[3,4]$.

Interestingly, in the low density regime, time-resolved luminescence (TR-PL) in QW is observed to be always dominated by light coming at the exciton energy, even under nonresonant excitation. The observations of this dominant contribution are so numerous that only a very partial list of references may be given here [5-9] (in order to be specific, we only consider here the case of QW grown on GaAs substrates). A double question has then been debated for more than 10 years in the literature: first how do free-electron-hole pairs bind into excitons and second does indeed the PL at very short time proceed from bound excitons. A brief survey of the literature allows to find that experimentalists have reported formation times ranging from less than $10 \mathrm{ps}$ up to about $1 \mathrm{~ns}$ [7-10] and theoretical values range from $100 \mathrm{ps}$ [11-13] to over 20 ns [14]. Clearly, the origin of this spreading in the reported values lies in the poor sensitivity of the experiments used in general to probe the exciton formation process, except for the case of the recent terahertz absorption experiments [10]. On the theoretical side, binding of an electron-hole pair into an exciton requires, at low temperatures, the emission of an acoustic phonon, which brings long formation time due to the small coupling of acoustic phonons to excitons.

The long formation time of excitons, together with the observation of PL at the exciton energy at the shortest
PACS numbers: 78.67.De, 71.35.Cc, 71.35.Ee, 78.47.+p

times $[7,15]$ led Kira et al. [16] to introduce the idea that a free-electron-hole plasma, properly including Coulomb correlation effects, should give rise to PL at the exciton energy, without any exciton population. Although this proposed interpretation is currently questioned [17], the dominance of the excitonic transition at short times has not received a sensible explanation yet.

In the present work, we use a properly designed sample with a particularly high quality, together with a PL setup with improved sensitivity, to study the exciton formation and to give clues on the origin of luminescence in QW. We will show that the formation times of excitons depend on the excitation density as expected from theory. We will also evidence how a very small population of excitons has a large enough recombination rate to dominate the PL spectrum, even at the shortest times accessible in the present experiment.

We have selected a particular sample with a single $\mathrm{In}_{x} \mathrm{Ga}_{1-x} \mathrm{As} 80 \AA \mathrm{QW}(x=5 \%)$, because of its unequalled quality. This $\mathrm{QW}$ is embedded in the middle of a $\lambda$ GaAs layer ( $\lambda$ corresponds to the wavelength of the QW excitonic resonance), which was grown over a 10 period distributed Bragg reflector (DBR). This DBR allows to measure directly the absorption of the sample in the reflection configuration. It also increases the optical coupling of the QW, but does not disturb the shape of the observed PL spectrum, because the resonance mode has a spectral width of about $40 \mathrm{~nm}$. Such a DBR changes slightly the radiative properties of free carriers, but does not affect their relaxation properties which we are studying here. The spectrum was recorded with a chargecoupled device camera in $\mathrm{cw}$ and with a streak camera in the time-resolved experiment (resolution of $3 \mathrm{ps,} \mathrm{photon-}$ counting mode). The temporal resolution of the whole setup is limited to about $20-30 \mathrm{ps}$, because of the spectral resolution of $0.1 \mathrm{meV}$. We excite the sample at $\hbar \omega=$ $1.5174 \mathrm{eV}$ in the range $1.0-300 \mu \mathrm{W}$ (photon density $N_{\nu}=9 \times 10^{8}-3 \times 10^{11}$ photons $/ \mathrm{cm}^{2}$ per pulse; diameter 
of $85 \mu \mathrm{m}$ ). The sample was kept at $5.0 \mathrm{~K}$. More details about the sample and the experimental setup can be found in [18].

The high quality of the sample is evidenced through optical measurements. We do not observe any Stokes shift between the absorption and PL at $E_{1 s}, E_{2 s}$, and $E_{\text {plasma }}$ (Fig. 1). The observed decay time of the QW emission at $1.4823 \mathrm{eV}$ reaches $3.7 \mathrm{~ns}$ in the low density limit at $10 \mathrm{~K}$, which indicates the very low density of nonradiative recombination centers. The linewidth of the exciton is less than $1.0 \mathrm{meV}$ showing some homogeneous broadening. Excited heavy-hole exciton state $2 s$ is clearly seen at $1.4882 \mathrm{eV}$ at low temperatures in $\mathrm{cw}$ experiments [18]. The free carrier PL shows the expected high energy exponential tail, corresponding to the Boltzmann distribution of the carriers. It dominates over the $2 s$ excitonic emission for temperatures above $25 \mathrm{~K}$ in $\mathrm{cw}$, and at all times in TR-PL.

The main interest of the sample is that the transitions at above gap energies can be resolved at all times and densities. Typical TR-PL traces are shown in Fig. 2(a). The sufficient dynamical range allows us to measure the free carrier temperature directly from the spectrum for all times after $100 \mathrm{ps}$ delay. Although it is possible to fit the exponential decay to the slope of the plasma transition for $t<100 \mathrm{ps}$ [these results are also shown in Fig. 2(b)] we think that it might not correspond to the real temperature. First, the diffused light of the excitation pulse at $t=0$ changes the shape of the spectrum. Second, the cooling process within first $30-40$ ps is very fast $[19,20]$ and due to the finite temporal resolution of our setup is not possible to observe it.

The cooling of carriers is modeled by computing the average energy-loss rate per electron-hole pair $\langle d E / d t\rangle \propto$ $\langle d T / d t\rangle$ and deducing the variations of their temperature from

$$
T(t)=T_{0}-\int_{0}^{t}\left\langle\frac{d T}{d t}\right\rangle d t
$$

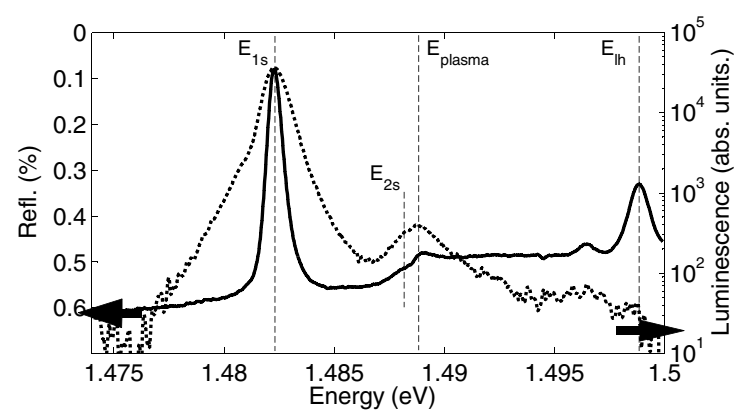

FIG. 1. cw absorption (i.e., 1 - reflectivity; bold line, left axis) and the TR-PL integrated over 1300 ps (logarithmic scale, right) results. $E_{1 s}=1.4823 \mathrm{eV}\left(E_{2 s}=1.4882 \mathrm{eV}\right)$ is the $1 s(2 s)$ heavy-hole exciton, $E_{\text {plasma }}=1.4888 \mathrm{eV}$, lighthole exciton $E_{\mathrm{lh}}=1.4988 \mathrm{eV}$ (vertical lines). The low energy exponential tail of the excitonic transition originates from the trion at $1.4807 \mathrm{eV}$ (discussed in [18]). where $T_{0}$ is the carrier temperature at $t=0$. Knowing the theoretical curve $\langle d E / d t\rangle$ for a Boltzmann-Maxwell distribution assuming a time-dependent temperature of bulk GaAs $[19,20]$ one can easily calculate the cooling curve by numerical integration with the only parameter $T_{0}$. However, in the case of QWs an ad hoc additional factor $\alpha>1$ is added $\langle d E / d t\rangle_{\text {exper }}=\langle d E / d t\rangle_{\text {theor }} / \alpha$ by which the measured energy-loss rate is reduced compared to the theoretical value [19-21]. Here we obtain a good fit for $\alpha=2.9$ [the bold line in Fig. 2(b)].

Very importantly, the shape of the PL signal above the gap is the same within the two main theoretical descriptions (excitons plus free carriers [6,22], or Coulomb correlated free carriers [16]). In both cases, we expect a Boltzmann like PL line provided electrons and holes are thermalized, which is the case for times longer than 100 ps. As will be shown below the PL of free carriers above the band edge provides a direct measure of the relative variations of the population of free electrons and holes. Indeed, in both models, the intensity at each energy is simply proportional to the product of the associated distribution functions $f_{e}$ and $f_{h}$. Then, in the case of the low density regime we are studying, the integrated intensity of the free carrier PL is proportional to the concentration of the electrons $n$ and holes $p$ via the bimolecular recombination rate $B$ [23], noting that $n=p$.

$$
I_{\text {plasma }} \sim B n p=B n^{2}
$$

$B$ is inversely proportional to the carriers" temperature $T$ at low temperatures in QW [24]:
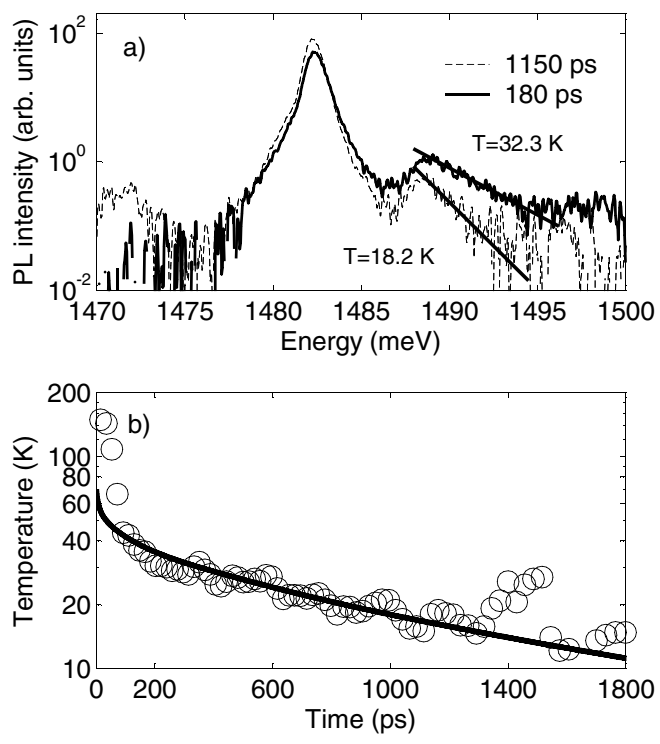

FIG. 2. (a) Spectral trace of the TR-PL $N_{\nu}=2 \times$ $10^{10}$ photons $/ \mathrm{cm}^{2}$. The temperatures of the carriers estimated 180 and 1150 ps after the excitation are shown. (b) The evolution of the carriers" temperatures deduced from the exponential slope of the PL above gap. The full line corresponds to the theoretical cooling curve. 


$$
B=\frac{8 \pi e^{2} n_{r}}{m_{0}^{2} c^{3}\left(m_{c}+m_{v}\right)}\left\langle\left|P_{c v}\right|^{2}\right\rangle \frac{E_{g}}{k_{B} T},
$$

where $n_{r}$ is the refractive index, $c$ is the light velocity, $m_{0}$ is the free-electron mass, $m_{c}$ and $m_{v}$ are the reduced electron and hole masses, $\left\langle\left|P_{c v}\right|^{2}\right\rangle$ is the momentum matrix element between electron and hole averaged over directions and light polarizations and over spins, $E_{g}$ is the energy gap in QW [24]. Equations (2) and (3) lead to

$$
n \propto \sqrt{I_{\text {plasma }} T} .
$$

Thus knowing the time evolution of both quantities - the free carriers PL intensity $I_{\text {plasma }}(t)$ and their temperature $T(t)$ [Eq. (1)] - we can deduce in a very simple and direct way the temporal evolution of the photoexcited free carrier density. This evolution is plotted in Fig. 3 (symbols) and is obtained from the results shown in Fig. 2 [25]. Comparing the relative values of carriers' and photons' densities it is clear that carriers are missing at early times in the high density experiments. We will show in the following that this is a direct signature of fast exciton formation.

This temporal evolution is governed by four factors: the electron-hole bimolecular recombination rate $B$, the nonradiative decay time $\tau_{\mathrm{nr}}$ and the formation and ionization of the excitons - via the bimolecular formation coefficient $C$. We get two rate equations for the population of free carriers $n$ and of excitons $X$ :

$$
\begin{aligned}
& \frac{d n}{d t}=-\gamma C n^{2}+\gamma C N_{\mathrm{eq}}^{2}-\frac{n}{\tau_{\mathrm{nr}}}-B n^{2}, \\
& \frac{d X}{d t}=\gamma C n^{2}-\gamma C N_{\mathrm{eq}}^{2}-\frac{X}{\tau_{\mathrm{D}}},
\end{aligned}
$$

$\tau_{\mathrm{D}}$ is a thermalized exciton decay time which depends on the fraction of excitons in the radiative region only. It

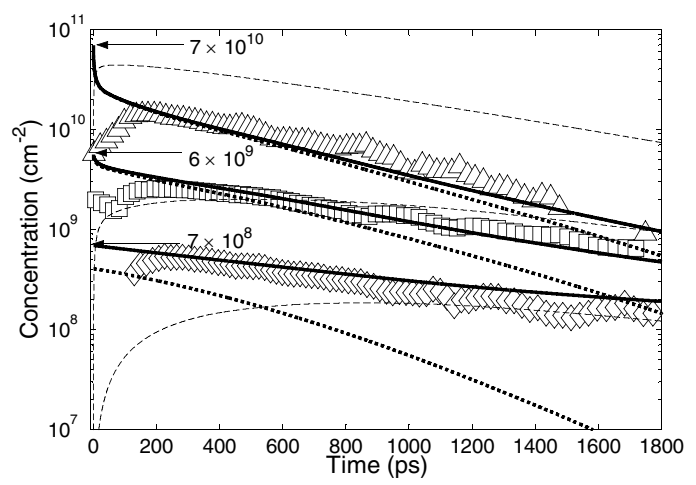

FIG. 3. Concentration of free carriers as a function of time. The experimental data calculated according to Eq. (4) for three different absorbed photon densities (given per pulse per $\mathrm{cm}^{2}$ ) are shown together with the time decay fitting curves [solid lines, Eq. (5)]. The corresponding $N_{\text {eq }}$ [Eq. (6)] are shown as the thick dotted lines; the total (i.e., radiative and dark) exciton density $X$ are marked as thin dashed lines. might be calculated according to [12] however, in order to reduce the number of free fitting parameters, we took a reasonable constant value $\tau_{\mathrm{D}}=700 \mathrm{ps}$ (to obtain the best fit shown in Fig. 4). $C$ is the rate calculated by Piermarocchi et al. [13]. It depends upon both carrier and lattice temperature through the interaction with optical and acoustic phonons. $\gamma$ is a multiplication factor by which the measured formation rate in our InGaAs QW is changed compared to the theoretical value obtained for GaAs. $N_{\mathrm{eq}}(n, X, T)$ is the equilibrium carrier concentration given by the Saha equation [26]:

$$
\frac{N_{\mathrm{eq}}^{2}}{X}=K(T)=\frac{\mu_{X} k_{B} T}{2 \pi \hbar^{2}} \exp \left(\frac{-E_{b}}{k_{B} T}\right),
$$

where $\mu_{X}$ is the reduced mass of the exciton and $E_{b}$ is the exciton binding energy and $n=p=N_{\mathrm{eq}}$. The term $C N_{\mathrm{eq}}^{2}$ describes exciton ionization. It ensures that the static solutions of Eq. (5) are the equilibrium values given by Eq. (6). This term corresponds to a first order approach of the rate equations valid in case the initial condition is a population of free carriers.

Having a sample of unprecedented quality with lifetimes in excess of $3 \mathrm{~ns}$, we can safely neglect the nonradiative processes $\tau_{\mathrm{nr}}$. The radiative decay of the electron-hole plasma is governed by $B$. The initial concentration is given by the absorbed photon density which can be estimated from the excitation photon density $N_{\nu}$ and the value of the absorption coefficient (Fig. 1). In our case, the InGaAs QW absorbs about 30\% of incoming photons at $1.5174 \mathrm{eV}$. Using the value of $B$ measured and computed by Matsusue et al. $B \approx 10^{-3} \mathrm{~cm}^{2} / \mathrm{s}$ at $15 \mathrm{~K}$ [24] and taking into account that the DBR shortens radiative times by about a factor of 10 , we get typical radiative decay times $(B n)^{-1}>10 \mathrm{~ns}$ for all the densities and temperatures of our experiment. Thus the dominant term in Eq. (5a) comes from the formation of excitons.

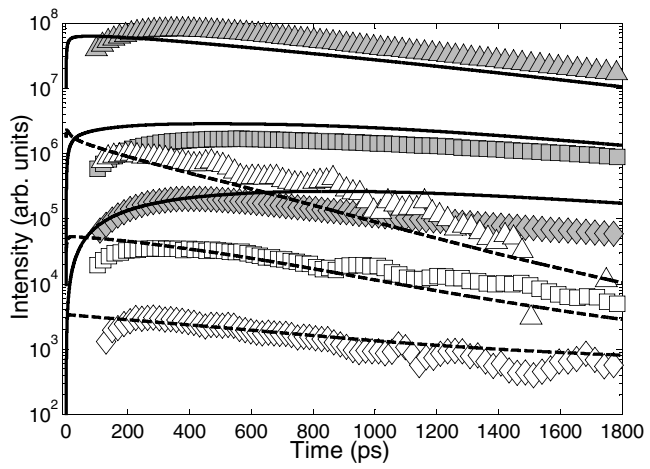

FIG. 4. Calculated intensity of the PL according to the results of the fit shown in Fig. 3 for excitons (solid lines, $I_{X}=X / \tau_{D}$ ) and free carriers (dashed lines, $I_{\text {plasma }}=B n^{2}$ ). The spectrally integrated experimental data $\left(I_{X}: 1.478-1.486 \mathrm{eV}\right.$, gray symbols; $I_{\text {plasma }}: 1.487-1.495 \mathrm{eV}$, open symbols) are for the excitation conditions used for Fig. 3. The important parameters here are $\tau_{D}=700 \mathrm{ps}$ and $B=0.01 \mathrm{~cm}^{2} / \mathrm{s}$ at $15 \mathrm{~K}$. 
In Fig. 3 the results of our model calculations are shown as solid lines. We used the values of the initial carrier concentration estimated from the number of absorbed photons as above. The time dependence of the temperature was taken from the experiment [Fig. 2(b)]. The only fitting parameter was $\gamma=3.5$.

The presented model includes all important ingredients of what should be a full theory, and follows the description proposed by Piermarocchi $[12,13]$. Very importantly, increasing the carrier density leads to a faster formation of excitons, because it gives rise to an increased probability of binding one electron and one hole through interaction with phonons. This theoretical prediction is indeed confirmed in our experiment. The formation time $\tau_{f}:=(\gamma \mathrm{Cn})^{-1}$, measured over 2 orders of magnitude in density 100 ps after the initial excitation, changes from less than $10 \mathrm{ps}$ for highest density to $570 \mathrm{ps}$ for the lowest one. This $\tau_{f}$ evolves as the plasma concentration and temperature change and, $1 \mathrm{~ns}$ after the excitation, binding of free carriers into excitons may be as long as 1100 ps. Thus the spread of the experimental values of $\tau_{f}$ found in the literature [7-10] might simply be related to the different experimental conditions.

This description also brings along an initial drop of the carrier concentration because of rapid exciton formation. Figure 3 indeed shows that, within the first $5 \mathrm{ps,} \mathrm{the}$ carrier concentration drops by about $40 \%$ for the highest densities. Such a rapid downfall is the consequence of the exciton formation through emission of LO phonons by the initially hot carriers [13]. This process is very efficient at high electron temperatures and densities. Our model includes this effect through an increase of $C$ with temperature and the linear dependence of the formation rate on the carrier density [Eq. (5), [13]]. Thus a considerable fraction of an initially hot free carrier population can be transformed into excitons during the first picoseconds. However, due to the large difference between the radiative rates of excitons and free carriers, the exciton contribution always dominates over that of free carriers. This is shown in Fig. 4 where we show the computed luminescence for free carriers and excitons deduced from the results shown in Fig. 3 together with experimental data. The spectrum is exciton dominated not only for higher excitations when the exciton formation is fast, but also in the case of low excitation density, due to the very small value taken then by the bimolecular recombination rate. The dominance of the free carrier PL over exciton PL would only occur for densities less than $10^{6} \mathrm{~cm}^{-2}$, a density never reached in time-resolved experiments.

It should be emphasized that we do not assume thermodynamical equilibrium between free carriers and excitons to obtain results presented in Fig. 3. The free carrier population (full line) is most of the time larger than the equilibrium solution (dotted line) due to the long exciton formation time at low densities. In fact, except for the higher densities, the process of exciton formation is so slow compared to the radiative decay of excitons, that the system is never in thermodynamical equilibrium. And even the top curve of Fig. 3 shows that, as the density lowers, the formation rate of excitons decreases and the Saha equilibrium cannot be sustained.

In summary, we have described here the results of the TR-PL study of a very high quality InGaAs QW sample. It has been possible to perform this experiment with a spectral resolution and a sensitivity allowing us to keep track of the separate excitonic and free carrier contribution over the whole time and density range. We have determined the temperature of the carriers and were able to deduce the formation time of excitons. The combination of our measurements and our modelization allows explaining in simple terms why the exciton luminescence dominates the spectrum at all times for all realistic pumping intensities used in time-resolved experiments.

This work was partially supported by the Swiss National Research fund. We wish to thank T. Guillet, J.-D. Ganiere, S. Koch, V. Savona, D. Chemla, and R. Zimmermann for fruitful discussions.

[1] D. A. B. Miller et al., Appl. Phys. Lett. 41, 679 (1982).

[2] C. Weisbuch et al., Solid State Commun. 37, 219 (1981).

[3] V. M. Agranovich et al., JETP Lett. 3, 223 (1966).

[4] B. Deveaud et al., Phys. Rev. Lett. 67, 2355 (1991).

[5] B. Deveaud et al., Appl. Phys. Lett. 51, 828 (1987).

[6] H.W. Yoon et al., Phys. Rev. B 54, 2763 (1996).

[7] T. C. Damen et al., Phys. Rev. B 42, 7434 (1990).

[8] D. Robart et al., Solid State Commun. 95, 287 (1995).

[9] B. Deveaud et al., J. Phys. IV 3, 11 (1993).

[10] R. A. Kaindl et al., Nature (London) 423, 734 (2003).

[11] A. Thilagam and Jai Singh, J. Lumin. 55, 11 (1993).

[12] C. Piermarocchi et al., Phys. Rev. B 53, 15834 (1996).

[13] C. Piermarocchi et al., Phys. Rev. B 55, 1333 (1997).

[14] W. Hoyer et al., Phys. Rev. B 67, 155113 (2003).

[15] G. R. Hayes et al. Phys. Status Solidi (a) 190, 637 (2002).

[16] M. Kira et al., Phys. Rev. Lett., 82, 3544 (1999); M. Kira et al., Phys. Rev. Lett., 81, 3263 (1998); S. Chatterjee et al., Phys. Rev. Lett., 92, 067402 (2004).

[17] K. Hannewald et al., Phys. Rev. B 62, 4519 (2000).

[18] J. Szczytko et al., Phys. Status Solidi (c) 1, 493 (2004).

[19] J. Shah, Ultrafast Spectroscopy of Semiconductors and Semiconductor Nanostructures, Solid State Sciences 115 (Springer, New York, 1996), pp. 161-192.

[20] K. Leo et al., Phys. Rev. B 38, 1947 (1988).

[21] W.W. Rühle et al., Phys. Rev. B 40, 1756 (1989).

[22] B. K. Ridley, Phys. Rev. B 41, 12190 (1990).

[23] G. Lasher and F. Stern, Phys. Rev. 133, A553 (1964).

[24] T. Matsusue et al., Appl. Phys. Lett. 50, 1429 (1987).

[25] For $I_{\text {plasma }}$ we integrated the intensity of $1.487-1.495 \mathrm{eV}$.

[26] R. T. Philips et al., Solid State Commun. 98, 287 (1996). 